\title{
Spontaneous Celiac Artery Dissection Presenting With Splenic Infarction: A Case Report
}

\author{
Ashraf Abugroun ${ }^{\mathrm{a}, \mathrm{b}}$, Arjun Natarajan ${ }^{\mathrm{a}}$, Hussein Daoud ${ }^{\mathrm{a}}$, \\ Habeeb Khalaf ${ }^{a}$
}

\begin{abstract}
Spontaneous isolated celiac artery dissection (SICAD) is extremely rare. We herein report a case of a 43-year-old male with no known history of cardiovascular disease who presented to the emergency department (ED) with sudden onset severe epigastric abdominal pain without a known trigger. Abdominal computed tomography (CT) scan showed a focal dissection and irregular enhancement of the celiac trunk with associated splenic artery embolus and large splenic infarct. The patient was successfully treated conservatively with blood pressure control, antiplatelet and anticoagulation therapy.
\end{abstract}

Keywords: Celiac artery dissection; Splenic infarction; Hypertension

\section{Introduction}

Spontaneous isolated celiac artery dissection (SICAD) is a rare entity. The first report of isolated celiac artery dissection was in 1947 [1]. Recent advances and wide use of imaging technologies allowed for early detection and timely management of such cases. More cases have been reported in the literature with wide variation in natural course and outcomes. To date, there is no consensus on the optimal management approach. In this case, we describe a 43-year-old male who presented with acute severe epigastric pain. Further imaging confirmed a diagnosis of celiac artery dissection and splenic infarction. The patient was successfully treated with adequate blood pressure control, antiplatelet and anticoagulation therapy. Follow-up imaging 6 months later showed adequate flow along the celiac artery with complete resolution of symptoms.

Manuscript submitted July 3, 2018, accepted August 8, 2018

aDepartment of Internal Medicine, Advocate Illinois Masonic Medical Center, 836 W Wellington Ave., Chicago, IL 60657, USA

bCorresponding Author: Ashraf Abugroun, Department of Internal Medicine, Advocate Illinois Masonic Medical Center, 836 W Wellington Ave., Chicago, IL 60657, USA. Email: Ashraf.abugroun@advocatehealth.com

doi: https://doi.org/10.14740/gr1065w

\section{Case Report}

A 43-year-old male with a past medical history of achalasia presented to the emergency department (ED) with sudden onset severe epigastric abdominal pain. The pain was constant, sharp, well localized, and lasted for several hours prior to admission without improvement. He had no nausea or vomiting and no change in bowel habits or stool. On arrival to the ED, he was afebrile, blood pressure (BP) was 142/82 $\mathrm{mm} \mathrm{Hg}$, and heart rate (HR) 60 beats per minute. Physical examination showed a soft non-distended abdomen without tenderness. He had positive bowel sounds. Labs showed a normal complete blood count (CBC), basal metabolic panel (BMP) and normal coagulation studies. Computed tomography (CT) scan of the abdomen and pelvis revealed a focal dissection and irregular enhancement of the celiac trunk with associated splenic artery embolus and large splenic infarct (Fig. 1,2). The radiological findings were consistent with celiac artery dissection. Transthoracic echocardiogram (TTE) showed normal ejection fraction, normal valves and no evidence of thrombus or vegetations. The patient's condition was managed medically. $\mathrm{He}$ received intravenous heparin, hydration, and analgesia. Blood pressure was controlled by the addition of carvedilol $3.25 \mathrm{mg}$ twice daily targeting a systolic blood pressure reading less than $110 \mathrm{~mm} \mathrm{Hg}$ (Fig. 3). The patient was discharged on warfarin for a total duration of 6 months with a target INR of 2 - 3. On outpatient follow-up, patient blood pressure was controlled and remained below 120/80 mm Hg and accordingly carvedilol was discontinued. Vascular aorta DPLEX performed 6 months following discharge showed patent superior mesenteric artery and celiac artery, with normal flow resistance waveforms and velocities within normal limits. Further hypercoagulability workup was not performed since the patient was lost to follow up.

\section{Discussion}

SICAD is a rare entity, typically iatrogenic or secondary to atherosclerosis, trauma, pregnancy, fibromuscular dysplasia, inflammatory or infectious diseases [2]. It is more common in men (5:1), and the mean age of patients is 55 years [3]. Predisposing factors as noted above tend to cause weakening of the arterial wall [3]. In our patient, no identifiable risk factor for SICAD was identified except for high blood pressure 


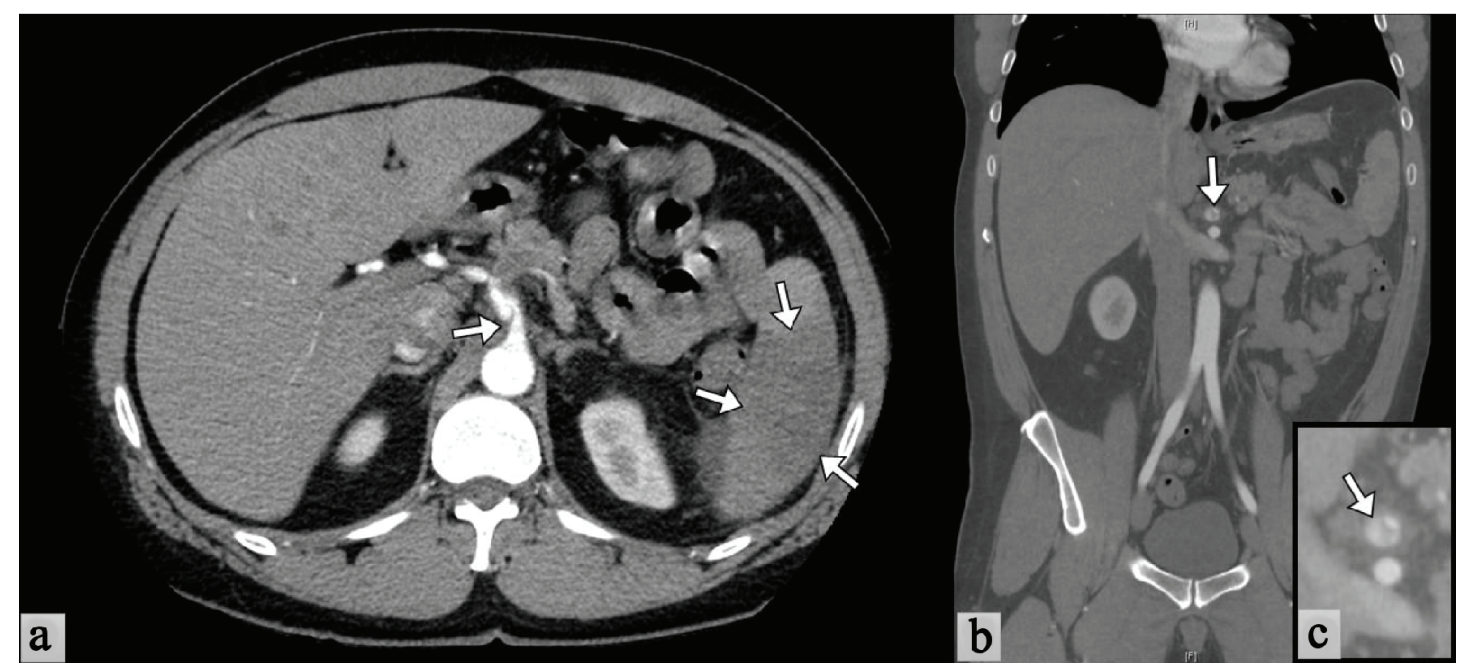

Figure 1. Abdominal CT scan with contrast. (a) Cross-sectional view shows a linear defect with mild dilation seen in the proximal celiac artery (arrow) compatible with acute vessel dissection. Thrombus in the distal celiac artery extending into the splenic artery with resultant splenic infarct (arrows). (b) Coronal view showing dissection along the origin of the celiac artery. (c) Magnified view of the area of dissection.

on admission. It was proposed that untreated hypertension might have contributed to the development of an intimal flap, which caused thrombosis and embolization downstream to the spleen. However, following discharge, patient blood pressure remained fairly controlled even without antihypertension medication use suggesting that high blood pressure readings during admission were likely related to physiologic response to pain.

Majority of patients who develop SICAD are asymptomatic due to lack of involvement of small bowel, as opposed to patients with splenic, renal or superior mesenteric artery involvement. The latter tend to suffer infarcts and bowel ischemia. Patients with chronic dissection, however, can present with intestinal angina and weight loss [4]. Commonly associ-

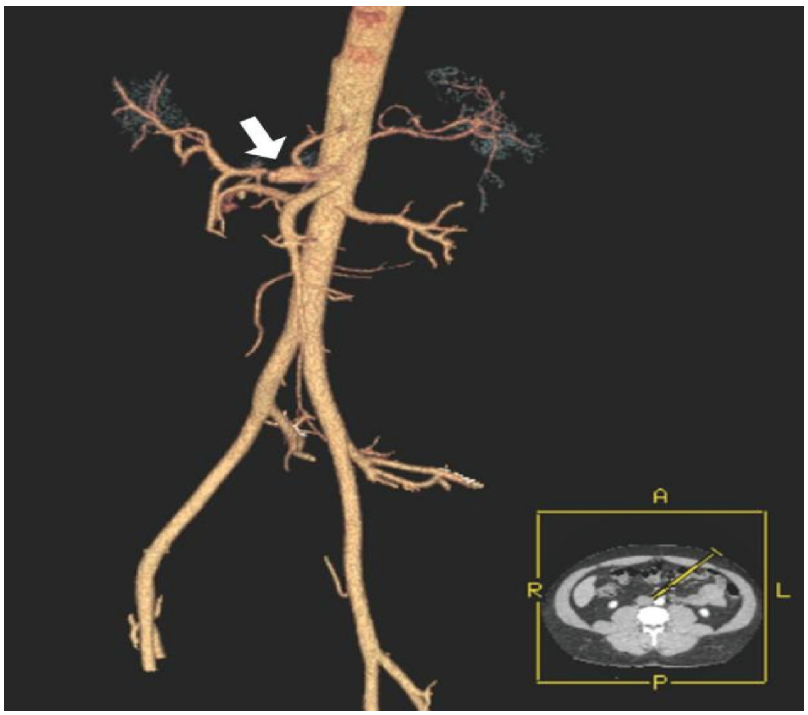

Figure 2. CTA of the abdominal aorta with 3-D reconstruction showing an area of aneurysmal dilation and dissection close to the origin of the celiac artery. ated complications of SICAD include aneurysmal formation resulting in bleeding, as well as late thrombosis resulting in ischemia [4]. Our patient was atypical in that he presented with sudden onset, severe acute epigastric abdominal pain.

The diagnostic technique of choice is contrast-enhanced $\mathrm{CT} /$ computed tomography with angiography (CTA) because it can show luminal borders and extraluminal involvement in three dimensions [5]. However, magnetic resonance (MR) angiography, sonography, and even conventional angiography can also be used. CT/CTA can show an intimal flap, which is pathognomonic, or an eccentric mural thrombus in the lumen and aneurysmal dilatation of the vessel which is highly suspicious [3, 6]. CT/CTA morphological classification (unless showing a dissecting aneurysm) does not affect management [7].

Interestingly, there are no guidelines for treatment. Most cases, regardless of their morphological appearance on CTA, are conservatively managed with antihypertensive therapy, with or without antiplatelet and anticoagulation therapy [7]. Antiplatelet therapy is preferred in patients with stenotic lesions [8]. Among patients with symptomatic SICAD, anticoagulation for a minimum of 3 to 6 months is recommended for prevention of thromboembolic complications, in addition to strict blood pressure control. The recommended INR goal with warfarin is 2 - 3 [8]. If a need for anticoagulation exceeds 6 months, interventional measures should be considered as lifelong warfarin has no known benefit in these patients [9]. Among patients with underlying vasculitis, anti-inflammatory medications including glucocorticoids are used [10, 11].

Endovascular stenting and surgical revascularization are typically reserved for symptomatic patients with evidence of ischemia $[12,13]$. Surgery includes resection of the dissected segment with anastomosis or bypass. It also allows for biopsy of the affected artery if vasculitis is suspected [4]. Recent studies suggest asymptomatic patients may even be simply observed and followed up closely with imaging [14]. Further- 


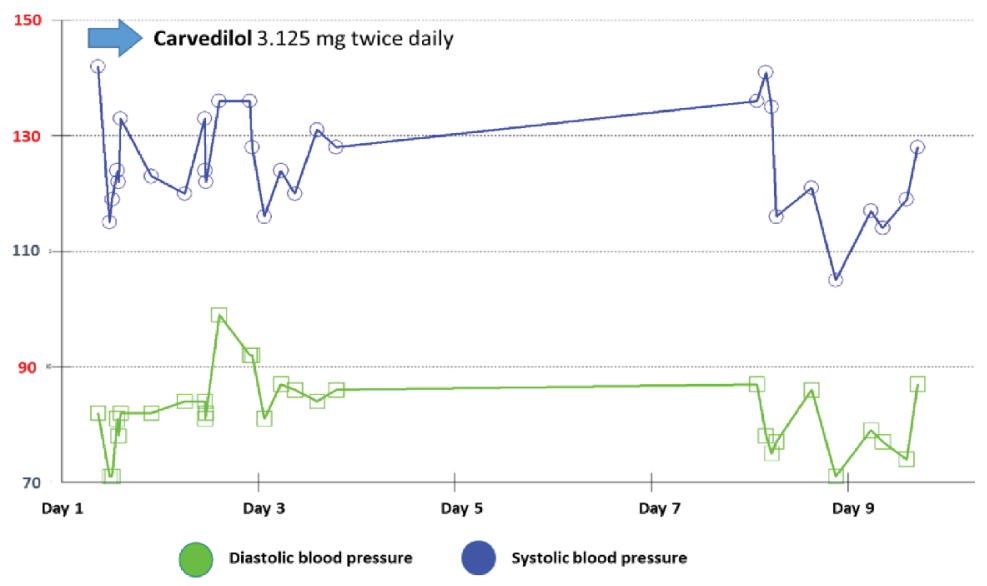

Figure 3. Blood pressure control during admission. Carvedilol $3.125 \mathrm{mg}$ (oral tabs) twice daily was initiated on admission day. Prior to discharge, a target systolic blood pressure below $110 \mathrm{~mm} \mathrm{Hg}$ was achieved.

more, they suggest symptomatic patients without evidence of ischemia may not require anticoagulation in addition to antiplatelet therapy [14].

\section{Conclusions}

SICAD is most often secondary to conditions which cause arterial wall weakening. Patients are usually asymptomatic; however, they may experience intestinal angina and weight loss or complications such as aneurysmal bleeding or ischemia secondary to a thrombus or embolus. SICAD has no universally agreed upon guidelines for treatment. Available therapeutic options include antiplatelet and anticoagulation therapy. Randomized controlled trials on large population samples are needed to define a more targeted treatment protocol.

\section{Acknowledgments}

Thanks to Dr. Vishnu Kuttappan from the Department of Radiology.

\section{Conflict of Interest}

None of the authors have any conflicts of interest to declare.

\section{Funding}

None of the authors have any source of funding to declare.

\section{References}

1. Bauersfeld SR. Dissecting aneurysm of the aorta; a presentation of 15 cases and a review of the recent literature. Ann Intern Med. 1947;26(6):873-889.
2. Fenoglio L, Allione A, Scalabrino E, Alberto G, Benedetti V, Pomero F, Valpreda S, et al. Spontaneous dissection of the celiac artery: a pitfall in the diagnosis of acute abdominal pain. Presentation of two cases. Dig Dis Sci. 2004;49(7-8):1223-1227.

3. D'Ambrosio N, Friedman B, Siegel D, Katz D, Newatia A, Hines J. Spontaneous isolated dissection of the celiac artery: CT findings in adults. AJR Am J Roentgenol. 2007;188(6):W506-511.

4. Glehen O, Feugier P, Aleksic Y, Delannoy P, Chevalier JM. Spontaneous dissection of the celiac artery. Ann Vasc Surg. 2001;15(6):687-692.

5. Subhas G, Gupta A, Nawalany M, Oppat WF. Spontaneous isolated superior mesenteric artery dissection: a case report and literature review with management algorithm. Ann Vasc Surg. 2009;23(6):788-798.

6. Kim JH, Roh BS, Lee YH, Choi SS, So BJ. Isolated spontaneous dissection of the superior mesenteric artery: percutaneous stent placement in two patients. Korean J Radiol. 2004;5(2):134-138.

7. Kim B, Lee BS, Kwak HK, Kang H, Ahn JH. Natural course and outcomes of spontaneous isolated celiac artery dissection according to morphological findings on computed tomography angiography: STROBE compliant article. Medicine (Baltimore). 2018;97(5):e9705.

8. Vaidya S, Dighe M. Spontaneous celiac artery dissection and its management. J Radiol Case Rep. 2010;4(4):3033.

9. Obon-Dent M, Shabaneh B, Dougherty KG, Strickman NE. Spontaneous celiac artery dissection case report and literature review. Tex Heart Inst J. 2012;39(5):703-706.

10. Takayama T, Miyata T, Shirakawa M, Nagawa H. Isolated spontaneous dissection of the splanchnic arteries. J Vasc Surg. 2008;48(2):329-333.

11. Poylin V, Hile C, Campbell D. Medical management of spontaneous celiac artery dissection: case report and literature review. Vasc Endovascular Surg. 2008;42(1):6264.

12. Choi JY, Kwon OJ. Approaches to the management of spontaneous isolated visceral artery dissection. Ann Vasc 
Surg. 2013;27(6):750-757.

13. Rama Krishnan R, Murali K, Madan R, Francis G. CT imaging findings and endovascular management of isolated spontaneous dissecting aneurysm of celiac artery. Indian J Radiol Imaging. 2013;23(3):234-237.
14. Loeffler JW, Obara H, Fujimura N, Bove P, Newton DH, Zettervall SL, van Petersen AS, et al. Medical therapy and intervention do not improve uncomplicated isolated mesenteric artery dissection outcomes over observation alone. J Vasc Surg. 2017;66(1):202-208. 